\title{
Respiratory syncytial virus
}

\author{
Benjamin G. Polkinghorne ${ }^{\mathrm{A}}$, Craig M. Mellis ${ }^{\mathrm{B}}$ \\ and Alison M. Kesson ${ }^{\mathrm{C}}$ \\ ${ }^{\mathrm{A}}$ NSW Public Health Officer Training Program, NSW Department \\ of Health \\ ${ }^{\mathrm{B}}$ Central Clinical School, Faculty of Medicine, The University \\ of Sydney \\ 'Infectious Diseases and Microbiology, The Children's Hospital \\ at Westmead
}

Respiratory syncytial virus (RSV) is the major viral respiratory pathogen of childhood, with approximately $95 \%$ of all children infected before 2 years of age. A United States study estimated RSV to be the most common cause of viral mortality in children aged under 5 years, but it was associated with substantially more deaths in the elderly (29.6 deaths per 100000 person-years). ${ }^{1}$ Infection is most common in winter in temperate climates and follows a distinctly seasonal pattern in New South Wales (NSW) with occasional epidemic years. The least RSV activity occurs in late January or early February while the peak occurs in early to mid-July each year. RSV is not notifiable in NSW and there is no specific public health response for sporadic infections.

\section{Acute viral bronchiolitis}

The most common presentation of RSV infection is acute viral bronchiolitis. While most infants with RSV-related bronchiolitis fully recover within 10-14 days, it is also the most frequent reason for hospitalisation of infants in developed countries. ${ }^{2}$

Bronchiolitis involves obstruction of the small, peripheral airways (bronchioles) due to the intense inflammation, mucosal oedema and sloughing or necrosis of the airway epithelium resulting from damage by the virus. Severely affected infants can present with symptoms including flared nostrils, rapid shallow breathing and retractions of the chest wall and muscles beneath the ribs as they struggle to inhale enough air. As well as cough, there is an audible 'wheezing' sound on expiration, and there are widespread inspiratory 'crackles' detectable by stethoscope. ${ }^{2}$

Infection with RSV provides only temporary immunity to disease upon reinfection, thus there is currently no vaccine available for RSV. Treatment is supportive and includes the provision of oxygen, fluids and close monitoring.

RSV is highly contagious and is transmitted by direct contact with nasal secretions or by fomite spread on hands, cots and fluffy toys. Preventing spread is possible by promoting cough etiquette, hand washing and cohorting hospitalised RSV patients. ${ }^{3}$ Palivizumab, a costly and controversial prophylaxis, has been shown to reduce hospitalisation if given monthly during RSV season. ${ }^{4}$

\section{Laboratory testing for RSV}

Rapid detection of RSV is useful to limit nosocomial spread and to implement timely treatment in severe cases. RSV antigen can be detected in a number of ways, including: viral culture; immunofluorescence; point of care tests (POCT); and nucleic acid tests. The presence of RSV antibody in blood can also be tested by serology. Optimal test selection depends on proper timing of specimen collection, type and quality of sample, patient age and specific assay. Specimens from the posterior nasopharynx are particularly desirable because they are likely to contain high titres of virus and large quantities of infected cells. Nasopharyngeal aspiration is more frequently performed on paediatric patients who are more tolerant of this procedure than older patients who prefer less invasive nasopharyngeal swabs.

Viral culture is slow and RSV can be difficult to grow; it can be useful when an isolate is needed for further characterisation. POCT are rapid and specific $(>90 \%)$ but sensitivity varies widely (60-90\% for RSV). Immunofluorescence has comparable sensitivity (80-95\%) and is often preferred over POCT because many viruses, including RSV, can be identified in approximately 1 hour. $^{5}$

Nucleic acid tests permit the widest array of respiratory viruses to be identified. They are also effective when specimen quality is compromised or when specimens were collected late in the illness. Sensitivity and specificity are equivalent or better than for other methods, but tests are slower and more expensive than other assays. Serology plays little role in the diagnosis of RSV, but it can be helpful for epidemiological purposes or to establish a retrospective diagnosis. ${ }^{5}$

\section{References}

1. Thompson WW, Shay DK, Weintraub E, Brammer L, Cox N, Anderson LJ et al. Mortality associated with influenza and respiratory syncytial virus in the United States. JAMA 2003; 289(2): 179-86. doi:10.1001/jama.289.2.179

2. Welliver RC. Review of epidemiology and clinical risk factors for severe respiratory syncytial virus (RSV) infection. $J$ Pediatr 2003; 143(5, Suppl): S112-7. doi:10.1067/S0022-3476(03) 00508-0

3. Groothuis J, Bauman J, Malinoski F, Eggleston M. Strategies for prevention of RSV nosocomial infection. J Perinatol 2008; 28(5): 319-23. doi:10.1038/jp.2008.37

4. The IMpact-RSV Study Group. Palivizumab, a humanized respiratory syncytial virus monoclonal antibody reduces hospitalization from respiratory syncytial virus infection in highrisk infants. Pediatrics 1998; 102(3): 531-7. doi:10.1542/ peds.102.3.531

5. Kesson AM. Respiratory virus infections. Paediatr Respir Rev 2007; 8(3): 240-8. doi:10.1016/j.prrv.2007.07.003 